\title{
Needle loss in subclavian vein during central venous catheter placement: case report of a rare complication
}

\author{
Daniela Botolin ${ }^{*}$, Annie Mooser ${ }^{2}$, Duane Stillions ${ }^{3}$, Keith Mortman $^{4}$, Shawn Sarin ${ }^{5}$ and Joseph Babrowicz ${ }^{6}$
}

\begin{abstract}
We present a case of needle separation during central venous catheter (CVC) placement in a super morbidly obese patient with subsequent surgical intervention in its retrieval. This complication, potentially lethal due to the relevant anatomy of such a procedure, alerts critical care physicians and surgeons to the possibility of equipment failure and stresses proper technique in what has become a routine procedure. It also emphasizes the routine use of ultrasound-guidance for cannulation in patients of any body habitus. While infection and arrhythmia are the generally known complications of CVC placement, clinicians must be alert to unanticipated events such as needle separation. In our case, the retrieval of this needle required multi-disciplinary intervention between radiology, critical care, vascular surgery, and thoracic surgery. Our event stresses hypervigilance to complications in a common procedure.
\end{abstract}

Keywords: Subclavian, Catheter, Fluoroscopy, Ultrasound, Obesity

\section{Background}

Within the critical care setting, CVCs are commonly used for intravenous therapy, dialysis, and the delivery of medications and nutrition, among other clinical interventions. CVC use was first documented by Werner Forssman, who as a surgical intern used his own cephalic vein to canalize his right atrium in 1929. In 1953, Seldinger perfected this technique [1]. In 1998, physicians in the United States inserted more than 5 million CVCs [2]. In 2011, 15 million catheter days, defined as the total number of days of patient exposure to CVCs, were documented in intensive care units across the country [3].

As with any procedure, proper precautions must be taken to avoid complications of CVC placement. With regard to catheters, this often emphasizes infection risk. There are complications of mechanical nature, as well. We report a rare event of needle separation during CVC insertion in the subclavian vein and the complexity of its removal.

\footnotetext{
* Correspondence: dbotolin@email.gwu.edu

'Department of Surgery, The George Washington University Hospital, 2150

Pennsylvania Avenue, NW, Suite 6B, Washington, DC 20037, USA

Full list of author information is available at the end of the article
}

\section{Case presentation}

A 46-year-old super morbidly obese male (BMI: 72) in renal failure requiring hemodialysis was admitted to the intensive care unit (ICU) following herniorrhaphy for an acute ventral hernia and incarcerated transverse colon. On post-operative day \#2, CVC placement into his left subclavian vein with an 18-gauge access needle of $6.35 \mathrm{~cm}$ length was attempted at the bedside in the intensive care unit. Due to the patient's excessive body mass it was necessary to use the entire length of the needle.

Following successful venous puncture with minimally applied pressure, the 18-gauge access needle fractured and separated from the hub device. At this time it was unknown if the foreign body was in the vascular space or in the left chest wall.

Initial attempt at retrieval took place at the bedside. The incision site was immediately extended in an attempt to track and retrieve the needle, which was palpated running parallel to the clavicle. Given the proximity to the great vessels and poor visualization, bedside intervention was stopped.

A portable AP chest $\mathrm{x}$-ray taken just after needle fracture showed a curvilinear density inferior to the left clavicle (Figure 1). 


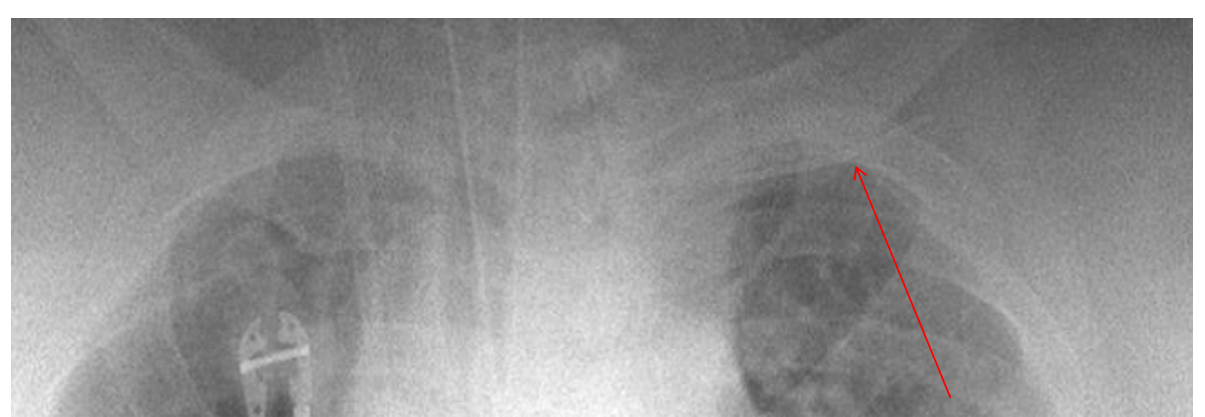

Figure 1 Portable AP Chest X-ray showing the fractured access needle within the left anterior chest wall.

On post-operative day \#3 the patient was taken to the operating room for needle retrieval under fluoroscopic guidance. The needle was visualized medial and deep to the previous chest wall incision. Muscles of the chest wall were divided and mobilized to expose the clavicle. Repeat fluoroscopy revealed the needle to be deep and medial to the costoclavicular ligament, approaching the chest (Figure 2).

To approximate the needle, the costoclavicular ligament was divided, and the subclavian vein was exposed. The original puncture site was encountered in the middle segment of the subclavian vein. Using this reference point, oblique images on the c-arm were then taken, and the retained needle was seen deep to the clavicle, still in the subclavian vein.

An endovascular approach was then used under fluoroscopy to retrieve the needle through the thoracoacromial vein. Ultimately, a snare device engaged the needle. The needle and snare were controlled with a sheath, and the needle was retracted successfully in one unit through the venous thoracoacromial branch (Figure 3).

\section{Conclusions}

Clinicians must be familiar with both the frequent and uncommon complications of this often used instrumentation. These complications have been divided into infectious, mechanical, and thrombotic categories [4]. Our event was mechanical in nature. According to past reviews, mechanical complications are more often associated with subclavian-site placement as compared to the internal jugular vein or femoral vein $[5,6]$. Specifically, compared to internal jugular CVC (35.9\% mechanical complication rate), subclavian sight complications are slightly higher at $39 \%$. Of these, arterial puncture, hematoma, and pneumothorax are most common [7]. There is no previous documentation to our knowledge of needle fracture at either an internal jugular, subclavian, or femoral site.

Unlike the technical error often associated with loss of the guide wire during catheterization [8] we attribute the access needle fracture to a faulty instrument. There was no deviation from standard technique; however, given the patient's body mass it is possible that our

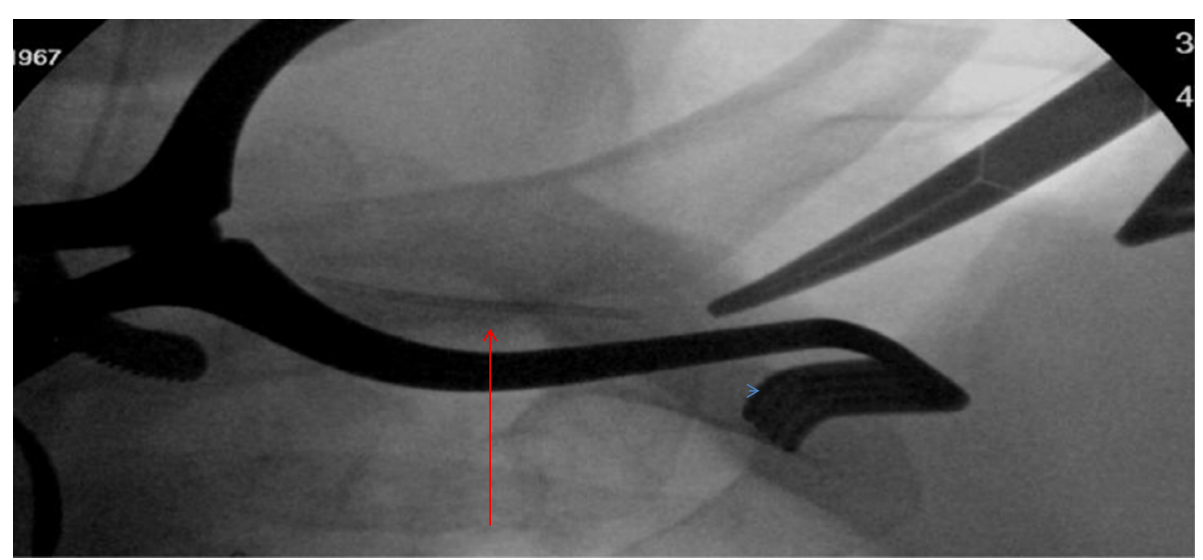

Figure 2 Intraoperative tonsil clamp and fluoroscopy indicated the needle positioned medial to the bedside incision, deep to the costoclavicular ligament. 


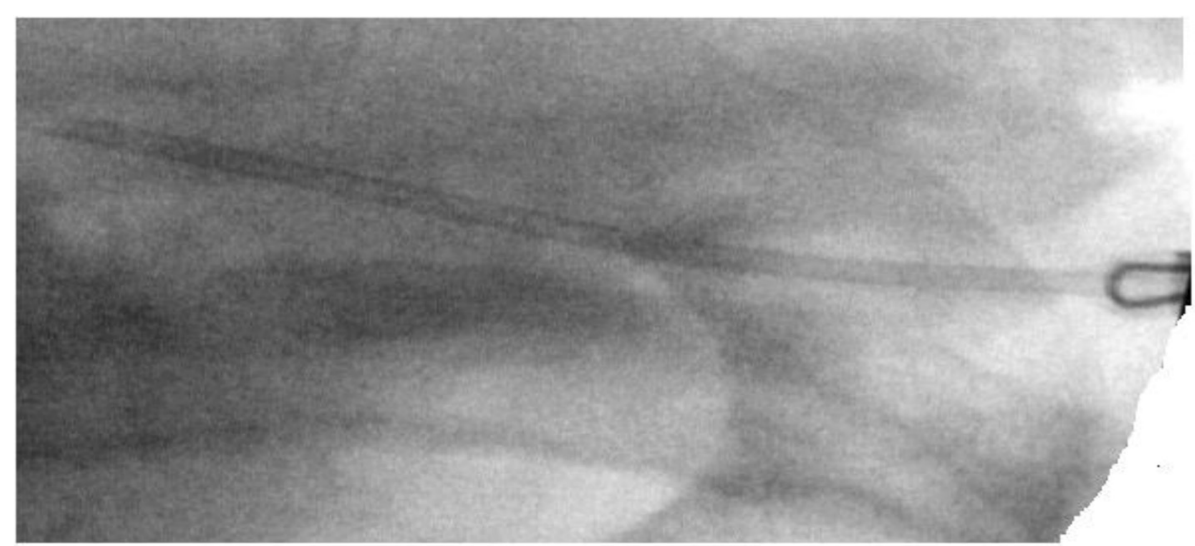

Figure $3 \mathrm{~A}$ micropuncture access set was used to puncture through the soft tissues on the lateral pectoral region into a thoracoacromial vein branch which had been exposed laterally on the subclavian vein. A snare device was passed through an 8-French sheath to engage and remove the needle.

needle bent from overlying pressure of the soft tissue without excessive applied force.

With regard to visualization during attempted access, we did not use ultrasound. In critical care patients, realtime ultrasound has been acknowledged for lower incidence of mechanical complications compared to the landmark method [9]. We were confident in infraclavicular positioning as an appropriate guide despite the patient's significant BMI. As stated above, we were able to palpate the needle superficially through the tissues after its initial detachment from the hub. Despite our confidence in positioning, retrospective discussion of our case highlights the importance of ultra-sound guidance in subclavian vein catheterization. Real-time ultrasound may have provided visual documentation of the needle bending under the stress of the patient's tissues prior to its departure from the hub.

While the consequences of needle fracture and loss within the vascular space have the potential for fatal complication, we were able to avoid hemodynamic compromise and lung injury by limiting our attempts for needle retrieval at the bedside. Our continued patient monitoring in the critical care setting and repeat chest $x$-ray ensured the stability of the fractured needle until operative intervention. The operating room is the optimal environment for intervention to retrieve an intravascular retained foreign body with such proximity to the great vessels.

Our case stresses that real-time ultrasound guidance in patients of any body habitus, including the super morbidly obese, should be standard of care. Technical difficulties include accurate probe placement and excessive infra-clavicular tissue obscuring angle of penetration. However, a clear two-dimensional image of the subclavian vein and overlying tissues may have provided visualization of the needle bending prior to this event and avoided necessity for such complicated retrieval.

\section{Consent}

Written informed consent was obtained from the patient's next of kin for publication of this case report and any accompanying images. A copy of the written consent is available for review by the Editor-in-Chief of this journal.

\section{Abbreviations}

CVC: Central venous catheter; BMI: Body Mass index; ICU: Intensive care unit; AP: Anterior-Posterior.

\section{Competing interests}

The authors declare that they have no competing interests.

\section{Authors' contributions}

DB AB-Drafted the manuscript, AM AB-Drafted the manuscript, DS FG-Helped to draft the manuscript, KM FG-Helped to draft the manuscript, SS FG-Helped to draft the manuscript, JB FG-Helped to draft the manuscript. All authors read and approved the final manuscript.

\section{Authors' information}

DB-MD, PGY 4, General Surgery, The George Washington University Hospital. AM-MD, PGY 1, General Surgery, Walter Reed National Military Medical Center. DS MD, MPH, Critical Care Fellow, The George Washington University Hospital. KM MD, FACS, FCCP, Director, Division of Thoracic Surgery, GWUH. Associate Professor, GWUSMHS. Member: Society of Thoracic Surgeons, Southern Thoracic Surgical Association, General Thoracic Surgical Club. SS MD, Vascular and Interventional Radiologist, GWUH. JB MD, Assistant Professor, GWUSMHS, Director of Vascular Surgery, Sibley Memorial Hospital.

\section{Author details}

'Department of Surgery, The George Washington University Hospital, 2150 Pennsylvania Avenue, NW, Suite 6B, Washington, DC 20037, USA. ${ }^{2}$ Division of General Surgery, Walter Reed National Military Medical Center, 8901 Rockville Pike, Arrowhead Building, 1st Floor, Bethesda, MD 20889, USA. ${ }^{3}$ Department of Anesthesiology and Critical Care Medicine, The George Washington University Hospital, 900 23rd Street, NW, Washington, DC 20037, USA.

${ }^{4}$ Division of Thoracic Surgery, The George Washington University Hospital, Foggy Bottom South Pavilion, 22nd \& I Street, NW, 6th Floor, Washington, DC 20037, USA. ${ }^{5}$ Department of Radiology, The George Washington 
University Hospital, 900 23rd Street, NW, Washington, DC 20037, USA.

${ }^{6}$ Division of Vascular Surgery, The George Washington University Hospital, Foggy Bottom South Pavilion, 22nd \& I Street, NW, 6th Floor, Washington, DC 20037, USA.

Received: 30 October 2014 Accepted: 16 December 2014

Published online: 24 February 2015

\section{References}

1. Seldinger SI. Catheter replacement of the needle in percutaneous arteriography; a new technique. Acta radiologica. 1953;39(5):368-76.

2. Raad I. Intravascular-catheter-related infections. Lancet. 1998;351(9106):893-8.

3. Casanova Vivas $\mathrm{S}$. Recommendations from CDC for the prevention of catheter-related infections (2013 update). Revista de enfermeria (Barcelona, Spain). 2014;37(4):28-33.

4. McGee DC, Gould MK. Preventing complications of central venous catheterization. New Eng J Med. 2003;348(12):1123-33.

5. Mansfield DC, Zeki SM, Mackenzie JR. Case report: extravasation of lipiodol-a complication of dacryocystography. Clin Radiol. 1994;49(3):217-8.

6. Randolph AG, Cook DJ, Gonzales CA, Pribble CG. Ultrasound guidance for placement of central venous catheters: a meta-analysis of the literature. Crit Care Med. 1996;24(12):2053-8.

7. Eisen LA, Narasimhan M, Berger JS, Mayo PH, Rosen MJ, Schneider RF. Mechanical complications of central venous catheters. J Intensive Care Med. 2006;21(1):40-6.

8. Schummer W, Schummer C, Gaser E, Bartunek R. Loss of the guide wire: mishap or blunder? British J Anaesth. 2002;88(1):144-6.

9. Fragou M, Gravvanis A, Dimitriou V, Papalois A, Kouraklis G, Karabinis A, et al. Real-time ultrasound-guided subclavian vein cannulation versus the landmark method in critical care patients: a prospective randomized study. Crit Care Med. 2011;39(7):1607-12.

\section{Submit your next manuscript to BioMed Central and take full advantage of:}

- Convenient online submission

- Thorough peer review

- No space constraints or color figure charges

- Immediate publication on acceptance

- Inclusion in PubMed, CAS, Scopus and Google Scholar

- Research which is freely available for redistribution 